\title{
Two N-Linked Glycans Differentially Control Maturation, Trafficking and Proteolysis, but not Activity of the IL-11 Receptor
}

\author{
Maria Agthe Yvonne Garbers $^{\mathrm{b}} \quad$ Joachim Grötzinger ${ }^{\mathrm{a}} \quad$ Christoph Garbers $^{\mathrm{a}}$ \\ aInstitute of Biochemistry, Kiel University, Kiel, 'DInstitute of Psychology, Kiel University, Kiel, Germany
}

\section{Key Words}

IL-11 • IL-11R • Gp130 • Glycosylation

\begin{abstract}
Background/Aims: The cytokine interleukin-11 (IL-11) has important pro- and antiinflammatory functions. It activates its target cells through binding to the IL-11 receptor (IL-11R), and the IL-11/IL-11R complex recruits a homodimer of glycoprotein 130 (gp130). $\mathrm{N}$-linked glycosylation, a post-translational modification where complex oligosaccharides are attached to the side chain of asparagine residues, is often important for stability, folding and biological function of cytokine receptors. Methods: We generated different IL-11R mutants via site-directed mutagenesis and analyzed them in different cell lines via Western blot, flow cytometry, confocal microscopy and proliferation assays. Results: In this study, we identified two functional N-glycosylation sites in the D2 domain of the IL-11R at N127 and N194. While mutation of N127Q only slightly affects cell surface expression of the IL-11R, mutation of N194Q broadly prevents IL-11R appearance at the plasma membrane. Accordingly, IL-11R mutants lacking N194 are retained within the ER, whereas the N127 mutant is transported through the Golgi complex to the cell surface, uncovering a differential role of the two $\mathrm{N}$-glycan sequons for IL-11R maturation. Interestingly, IL-11R mutants devoid of one or both $\mathrm{N}$-glycans are still biologically active. Furthermore, the IL-11RN127Q/N194Q mutant shows no inducible shedding by ADAM10, but is rather constitutively released into the supernatant. Conclusion: Our results show that the two $\mathrm{N}$-glycosylation sites differentially influence stability and proteolytic processing of the IL-11R, but that N-linked glycosylation is not a prerequisite for IL-11 signaling.
\end{abstract}




\section{Introduction}

The attachment of complex carbohydrate moieties to the side-chain of asparagine residues of proteins, hereafter referred to as $\mathrm{N}$-glycosylation, is a post-translational modification often essential for stability, folding and biological function of a given protein that is either secreted or membrane-bound $[1,2]$. This modification is quite common, and it was estimated that the majority of all proteins are in fact glycoproteins [3].

$\mathrm{N}$-glycosylation requires the presence of the consensus sequence motif (called sequon) Asn-X-Ser or Asn-X-Thr, where X denotes every amino acid residue except proline [4]. $\mathrm{N}$-glycosylation starts in the endoplasmic reticulum (ER) via the addition of a dolichol-linked $\mathrm{N}$-Acetylglucosamine monosaccharide. Afterwards, a pre-formed precursor oligosaccharide is transferred and the first trimming steps of this precursor occur. Final modifications to the oligosaccharide are made afterwards in the Golgi complex [4]. In contrast, the addition of smaller oligosaccharides to serine or threonine residues independent of a defined sequon, termed 0-glycosylation, occurs exclusively within the Golgi complex [5].

Interleukin-11 is a member of the IL-6 family of cytokines [6, 7]. It is a secreted glycoprotein that is rarely detectable in body fluids of healthy individuals, but its expression is enhanced in different human diseases, e.g. pancreatic cancer [8], breast cancer with bone metastasis [9], acute pancreatitis [10] and arthritis [11]. IL-11 causes preterm birth [12] and features of preeclampsia in mice [13]. Furthermore, IL-11 has been described as the key cytokine of the IL- 6 family in gastrointestinal tumorigenesis, thereby offering a potential therapeutic target [14].

IL-11 exerts its biological functions through binding to the IL-11 receptor (IL-11R), which is expressed on a variety of tissues, including bone, endometrium, liver, lung and the gastrointestinal tracts (reviewed in detail in $[7,15]$ ). The IL-11R is a type-I transmembrane protein, whose extracellular part consists of an Ig-like domain (called "D1"), two fibronectintype III-like domains ("D2" and "D3") and a flexible linker region ("stalk"). This ectodomain is followed by a membrane-spanning region and a short intracellular region [16]. Upon ligand binding, which is facilitated by the so-called cytokine-binding module located in D2/D3, a homodimer of the common $\beta$-receptor of the IL- 6 family, glycoprotein (gp)130, is recruited, and intracellular signaling cascades, among them the Janus kinase/Signal Transducer and Activator of Transcription (Jak/STAT) pathway, are activated [7, 17]. In polarized cells, the IL-11R localizes to apical and basolateral membranes and has transcytotic activity [18]. We have recently shown that the IL-11R can undergo limited proteolysis, predominantly by the metalloprotease ADAM10, and that the resulting soluble IL-11R (sIL-11R) can form an agonistic complex with its ligand IL-11 that is able to activate cells irrespective of their own IL-11R expression $[19,20]$. This process, which we have termed IL-11 trans-signaling, closely resembles the trans-signaling pathway of IL-6 [21]. Interestingly, IL-11 trans-signaling is dispensable for fertility and gastric tumor formation in mice [22, 23].

Previous work on gp130 revealed that a variant where all nine functional N-glycosylation sites were mutated was predominantly degraded via the proteasome [24]. The small proportion that was transported to the cell surface was biologically active, however, letting the authors conclude that N-glycosylation of gp130 was important for the stability, but not for its signaling capacity [24]. Furthermore, we have recently shown that $\mathrm{N}$-glycosylation of the IL-6 receptor (IL-6R) is dispensable for its biological function, but rather influences limited proteolysis of the IL-6R by the metalloprotease ADAM17 [25]. In contrast, the receptors for C-X-C motif chemokine 12 (CXCL12), granulocyte-macrophage colony-stimulating factor (GM-CSF), epidermal growth factor (EGF) or interleukin-5 require proper glycosylation for their biological functions [26-29].

A functional role of glycosylation for the biological function, stability and folding of the IL$11 \mathrm{R}$ has not been investigated so far. In this study, we identify two functional N-glycosylation sites in the D2 domain of the IL-11R as revealed by deglycosylation experiments and site-directed mutagenesis. We further show that these glycans are essential for proper expression of the receptor at the cell surface with a differential role for the two sequons in 
terms of receptor maturation. However, an IL-11R devoid of N-glycans is still able to induce phosphorylation of the transcription factor STAT3 and IL-11-dependent cell proliferation. However, we observed no inducible shedding by ADAM10 of this IL-11R mutant, suggesting that $\mathrm{N}$-glycosylation is not a prerequisite for IL-11 signaling, but rather influences stability and proteolytic processing of the IL-11R.

\section{Materials and Methods}

\section{Cells and Reagents}

HEK293 cells were from DSMZ GmbH (Braunschweig, Germany), Phoenix-Eco cells were provided by U. Klingmüller (DKFZ, Heidelberg, Germany) and Ba/F3-gp130 cells were described previously [30]. All cell lines were cultured under standard conditions in DMEM medium (Sigma Aldrich, Steinheim, Germany) supplemented with $10 \%$ fetal bovine serum, penicillin $(60 \mathrm{mg} / \mathrm{l})$ and streptomycin $(100 \mathrm{mg} / \mathrm{l})$. Cells were grown at $37^{\circ} \mathrm{C}$ and $5 \% \mathrm{CO} 2$ in a standard incubator with a water-saturated atmosphere. The culture medium for Ba/F3-gp130 additionally contained $10 \mathrm{ng} / \mathrm{ml}$ Hyper-IL- 6 which was expressed and purified as described in [31]. After stable transduction Ba/F3-gp130 IL-11R variant cells were cultured with $10 \mathrm{ng} /$ $\mathrm{ml}$ recombinant IL-11 instead of Hyper-IL-6, and IL-11 was produced as described [18, 32]. The anti-IL-11R pAb N-20 was obtained from Santa Cruz Biotechnology (Santa Cruz, CA, USA), the anti-GAPDH mAb, antimyc-tag mAb, anti-pSTAT3 and anti-STAT3 mAb were from Cell Signaling (Frankfurt/M, Germany) and the anti-GM130 mAb was from BD Bioscience. Peroxidase-conjugated secondary anti-rabbit and anti-mouse antibodies were purchased from ImmunoResearch Laboratories (Dianova GmbH, Hamburg, Germany). The AlexaFluor488-conjugated (anti-rabbit) and the AlexaFluor594-conjugated (anti-mouse) secondary antibodies were obtained from Immunotools (Thermo Scientific, Bonn, Germany). PNGaseF, EndoH and O-glycosidase used for deglycosylation (see below) were from New England Biolabs (Frankfurt/M, Germany). The ADAM10-selective inhibitor GI254023X was synthesized by Iris Biotech (Marktredwitz, Germany). MG132 and NH4Cl were purchased from Sigma-Aldrich (Steinheim, Germany).

\section{Construction of expression plasmids}

The expression plasmid pcDNA3.1-myc-hIL-11R containing the complete cDNA of the human IL-11R plus an $\mathrm{N}$-terminal c-myc tag has been described previously [16]. The IL-11R variants containing only one mutated $\mathrm{N}$-glycosylation sequon (N127Q and $\mathrm{N} 194 \mathrm{Q}$ ) were constructed via splicing by overlapping extension PCR and inserted into pcDNA3.1-myc-hIL-11R via two SacI restriction sites. The IL-11R double mutant (N127Q/N194Q) was constructed from both single mutants via two BamHI restriction sites. For stable transduction of Ba/F3-gp130 cells the receptor variants were subcloned into pMOWS plasmids via Pmel restriction sites.

\section{Retroviral transduction of Ba/F3-gp130 cells}

Stable transduction of Ba/F3-gp130 using a retroviral system was conducted as described in detail elsewhere [33]. Cells stably expressing the IL-11R variants were selected with puromycin $(1.5 \mu \mathrm{g} / \mathrm{ml})$ and further cultivated with recombinant IL-11 $(10 \mathrm{ng} / \mathrm{ml})$.

\section{Ectodomain shedding assay and lysis of cells}

HEK293 cells were transiently transfected with plasmids coding for the different IL-11R variants. 48 $\mathrm{h}$ after transfection cells were treated in serum-free medium with ionomycin $(1 \mu \mathrm{M})$ or GI254023X $(3 \mu \mathrm{M})$ for 1 or $4 \mathrm{~h}$, respectively. Cell culture supernatant and cells were then separately harvested and cell culture supernatants containing the soluble cytokine receptors were precipitated using $20 \%$ trichloracetic acid as described previously [19]. Cells were lysed in lysis buffer (50 mM Tris-HCl pH 7.5, $150 \mathrm{mM} \mathrm{NaCl}, 1 \%$ Triton-X 100 and Complete protease inhibitor cocktail (Roche Applied Science, Penzberg, Germany)) and subjected to a semi-dry Western blotting procedure on a $10 \%$ SDS gel and transferred onto a PVDF membrane.

Deglycosylation of IL-11R

$15 \mu \mathrm{g}$ protein from cell lysates of transiently transfected HEK293 cells or stably transduced Ba/F3gp130-IL-11R cells were denatured $\left(10 \mathrm{~min}\right.$ at $95^{\circ} \mathrm{C}$ ) and incubated with $1 \mu \mathrm{l}$ (500 units) of PNGaseF or 


\section{Cellular Physiology Cell Physiol Biochem 2018;45:2071-2085 and Biochemistry Published online: March 12, 2018 \begin{tabular}{l|l} 
DOI: 10.1159/000488044 & $\begin{array}{l}\text { C } 2018 \text { The Author(s). Published by S. Karger AG, Basel } \\
\text { www.karger.com/cpb }\end{array}$
\end{tabular}}

Agthe et al.: N-Glycosylation of the IL-11R

O-glycosidase overnight at $37^{\circ} \mathrm{C}$. The reaction mix was then boiled in $5 \mathrm{x}$ Laemmli buffer $\left(10 \mathrm{~min}\right.$ at $\left.95^{\circ} \mathrm{C}\right)$ and subjected to Western blot analysis.

\section{Flow cytometry}

In order to stain cell surface expression of different IL-11R variants, transiently transfected HEK293 cells and stably transduced Ba/F3-gp130-IL-11R cells were stained extracellularly with an anti-myc-tag $\mathrm{mAb}$ and a secondary AlexaFluor488-conjugated anti-rabbit antibody. A detailed staining protocol is described in [20]. Cells were analyzed on a BD FACS CantoII using the FACS Diva software and FCS Express (De Novo Software, Los Angeles, California, US).

\section{Immunofluorescent staining and confocal microscopy}

HeLa cells were seeded on coverslips and transiently transfected on the following day. $48 \mathrm{~h}$ after transfection cells were subjected to immunofluorescent staining. For extracellular staining only, cells were fixed with 4\% PFA at RT for $10 \mathrm{~min}$, incubated in 0.12\% glycin/PBS (10 min at RT) and blocked with 10\% fetal calf serum/PBS ( $1 \mathrm{~h}$ at RT). Cells were stained with anti-myc-tag mAb (1:200 in blocking solution) at RT for $1 \mathrm{~h}$, washed three times in PBS and incubated with AlexaFluor488-conjugated anti-rabbit IgG (1:300 in blocking solution) again for $1 \mathrm{~h}$ at RT. Coverslips were washed three times in PBS and mounted with ProLong Gold Antifade reagent containing DAPI (Invitrogen, Karlsruhe, Germany). For additional intracellular staining or the double staining with the Golgi marker GM130, cells were stained as described above but permeabilized by addition of $0.2 \%$ saponin to the blocking solution. Microscopic slides were analyzed on a confocal laser scanning microscope (Olympus FluowView FV1000) with a 60x oil-immersion objective (NA:1.35) at RT. The Manders' M2 Coefficient for co-localization of the IL-11R and GM130 was calculated via the Image J plugin JACoP (NIH, Bethesda, US).

\section{Ba/F3-gp130 cell proliferation assay}

Cell proliferation of the different Ba/F3-gp130-IL-11R cell lines in response to recombinant IL-11 was determined using the CellTiter Blue Viability Assay (Promega, Karlsruhe, Germany) following the manufacturer`s instructions. In short, equal numbers of Ba/F3-gp130 IL-11R cells were incubated with increasing amounts of IL-11 ( $0-100 \mathrm{ng} / \mathrm{ml})$ for $48 \mathrm{~h}$ at $37^{\circ} \mathrm{C}$. After addition of the reaction reagent the fluorescence intensity (RLU; relative light units) was measured at $t=60 \mathrm{~min}$ and normalized to the RLU obtained at $\mathrm{t}=0 \mathrm{~min}$.

\section{Detection of (p)STAT3 in Ba/F3-gp130-IL-11R cells}

The different Ba/F3-gp130-IL-11R cell lines were washed twice in PBS and serum-starved for $4 \mathrm{~h}$. Then, $1 \times 10^{6}$ cells were stimulated with recombinant IL-11 $(10 \mathrm{ng} / \mathrm{ml})$ for different time periods $(0-60$ $\min$ ) at $37^{\circ} \mathrm{C}$. To stop stimulation cells were centrifuged and boiled in $50 \mu \mathrm{l} 2.5 \mathrm{x}$ Laemmli buffer. $20 \mu \mathrm{l}$ of the cell lysate was loaded on a 10\% SDS gel, transferred onto a PVDF membrane, and this was used for detection of pSTAT3 and STAT3.

\section{Statistical analyses}

Numerical data were analyzed for statistical significance either via one-sample t-test or one-way ANOVA. Tukey's multiple comparison test was applied when appropriate. A p-value $<0.05$ was considered as statistically significant.

\section{Presentation of experimental data}

Data are presented as mean \pm SD from at least three independent experiments. For confocal images, Ba/F3-gp130 cell proliferation data and Western blots, one experiment from at least three with similar outcome is shown.

\section{Results}

Identification of two N-glycosylation sites within the D2 domain of the IL-11R

The IL-11R and the IL-6R show a similar modular organization consisting of three domains and regions which fulfill different functional roles [16]. Despite this, the crystal 
structure of IL-11 suggested structural differences from IL-6 [34], and we have recently shown that proteases differ in their ability to cleave IL-11R and IL-6R [19]. A functional role for N-glycans in IL-11R biology has not been reported so far, but $\mathrm{N}$-glycosylation is dispensable for the biological activity of the IL-6R, although it regulates ADAM17-mediated proteolysis [25].

$\mathrm{N}$-glycosylation of a protein can only occur if a specific sequon (Asn-X-Ser or Asn-X$\mathrm{Thr}$ ) is present. In agreement with a previous report [35], we identified two sequons containing the asparagine residues N127 and N194 within the D2 domain of the IL-11R that could be potentially used for N-glycosylation (Fig. 1A, B). We further performed an amino acid sequence alignment of the D2 domains of human, murine and rat IL-11R and found that these sequons are strictly conserved between the three mammalian species, suggesting that results obtained for the human IL-11R in this study could be transferred to their murine and rat homologs (Fig. 1C).

As the presence of a sequon does not guarantee that it will indeed be used for glycosylation, we sought to analyze whether this is the case. We transiently transfected HEK293 cells with a cDNA encoding N-terminal myctagged human IL-11R and lysed these cells $48 \mathrm{~h}$ after transfection and analyzed IL-11R expression via Western blot. As described [36], the IL-11R was present as at least three distinct bands of different molecular sizes (Fig. 1D, first lane). Furthermore, we incubated equal amounts of the cell lysate with the deglycosylating enzymes PNGaseF or O-glycosidase. PNGaseF is able to cleave complex, fully matured glycans as well as so-called "high mannose" glycans which are intermediates that occur during protein maturation in the ER and Golgi complex. As shown in Fig. 1D, incubation with PNGaseF led to a reduction of the apparent molecular mass of the IL-11R, and the strongest band observed corresponds to the unglycosylated IL-11R. Interestingly, a second band with higher molecular mass was also detected, suggesting that the IL-11R is not only N-glycosylated, but carries at least another

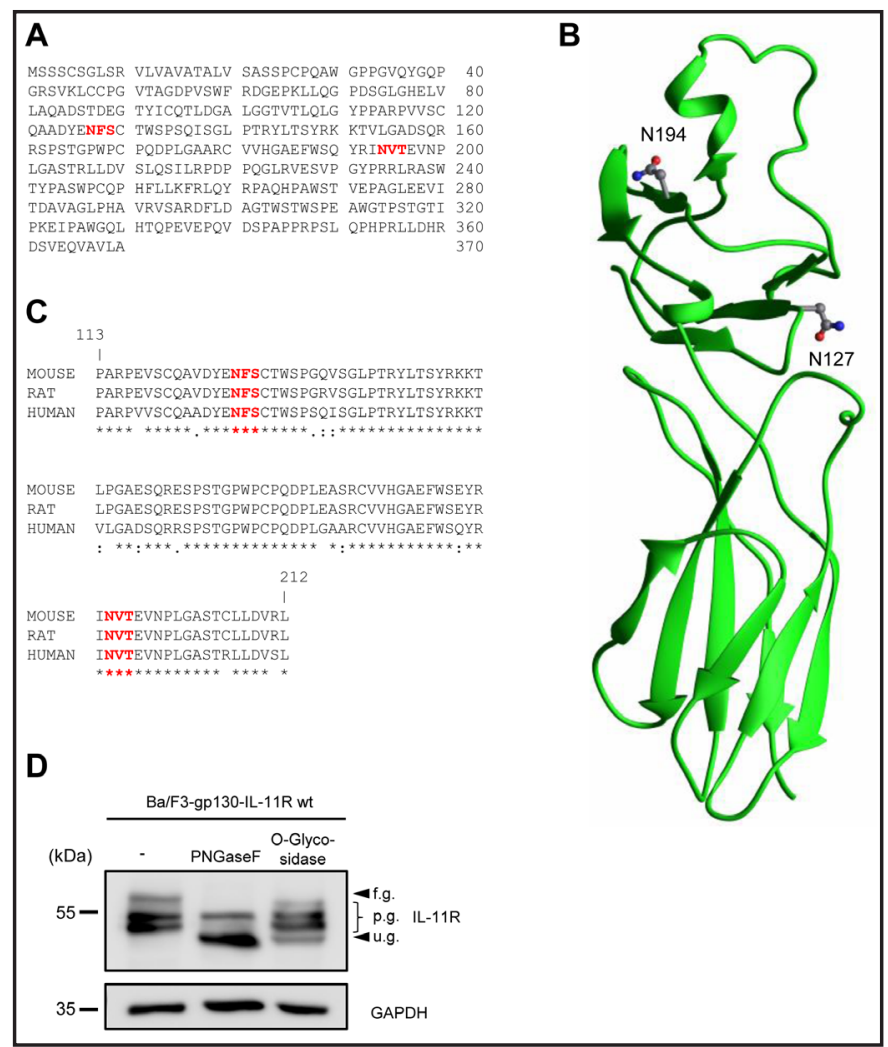

Fig. 1. The IL-11R contains two putative N-glycosylation sites. A: Amino acid sequence of the human IL-11R ectodomain (UniProt: Q14626). Highlighted in red are the two identified sequons (N-X$\mathrm{S} / \mathrm{T}$ ) that are potentially used for $\mathrm{N}$-glycosylation. B: Structural model of the two IL-11R fibronectin type III domains (D2 and D3). Indicated are the two asparagine residues N127 and N194 in D2. C: Sequence alignment of the D2 domain of murine, rat and human IL-11R. The identified sequons are shown in red. '*' indicates identical amino acids, ':' indicates amino acids with similar properties, ". indicates amino acids with weak similarity. D: HEK293 cells were transiently transfected with a plasmid lysed and treated with the deglycosylating enzymes PNGaseF and 0 -glycosidase over night at $37{ }^{\circ} \mathrm{C}$ or left untreated. IL-11R banding pattern was analyzed via Western blot. GAPDH served as loading control. One representative blot out of three independent experiments is shown. f.g.: fully glycosylated, p.g.: partially glycosylated, u.g.: unglycosylated. encoding the human IL-11R. $48 \mathrm{~h}$ after transfection cells were 
post-translational modification. Indeed, also treatment with 0 -glycosidase led to a shift in the band pattern, indicating that the IL-11R is also 0-glycosylated, a modification that can occur at any serine or threonine residue without the need of a specific sequon. In summary, these results show that at least one of the two sequons of the IL-11R is indeed used for N-glycosylation, and suggest that the IL-11R carries at least one 0-linked glycan.

Mutation of N127 and N194 results in an $I L-11 R$ devoid of $\mathrm{N}$-glycans

In order to investigate if one or both of the identified sequons are used for N-glycosylation, we constructed three different IL$11 \mathrm{R}$ mutants where either one or both asparagine residues were replaced by glutamine residues (termed IL-11R-N127Q, IL-11RN194Q and IL-11R-N127Q/ N194Q, respectively). We first expressed all three mutants

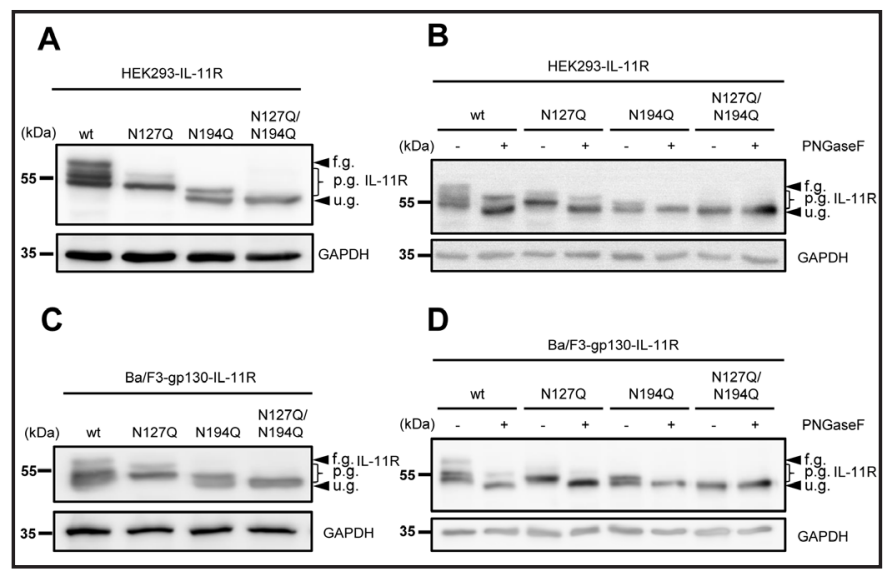

Fig. 2. N127 and N194 are the only two N-glycosylation sites used in the IL-11R. A: HEK293 cells were transiently transfected with expression plasmids coding for IL-11R wt or any of the three mutants N127Q N194Q or N127Q/N194Q as indicated. 48 $\mathrm{h}$ after transfection cells were lysed and analyzed via Western Blot. B: HEK293 cells were transfected as described in panel A. Cell lysates were then subjected to PNGaseF treatment over night at $37^{\circ} \mathrm{C}$ and IL-11R visualized via Western blot. C: Ba/F3-gp130 cells stably transduced with IL-11R wt, N127Q, N194Q or N127Q/ N194Q were lysed and analyzed for the expression of IL-11R via Western blot. D: Ba/F3-gp130 cell lysates were deglycosylated over night at $37^{\circ} \mathrm{C}$ using PNGaseF and the IL-11R visualized via Western blot. GAPDH served as control for equal protein loading. One representative blot out of three independent experiments is shown. f.g.: fully glycosylated, p.g.: partially glycosylated, u.g.: unglycosylated. and the IL-11R wt transiently in HEK293 cells and analyzed the resulting band pattern via Western blot analysis. As seen before (Fig. 1D), IL-11R wt showed three distinct bands of different molecular weight. In contrast, we observed only two different bands for both IL-11R-N127Q and IL-11R-N194Q, which had all a lower molecular weight than the upper band of IL-11R wt (Fig. 2A), which likely represents the fully glycosylated form (Fig. 1D, 2A). In contrast, IL-11R-N127Q/N194Q migrated as a single band (Fig. 2A). These results suggest that the different bands observed for IL-11R wt originate indeed from differently glycosylated intermediate forms, and confirm that the two sequons containing Asn-127 and Asn-194 are both used for N-glycosylation of the IL-11R. Interestingly, the band patterns observed in HEK293 cells transfected with either IL-11R-N127Q or IL-11R-N194Q were not identical, suggesting that the two sequons are not used equally during IL-11R maturation.

We further sought to investigate these differences between the two sequons and therefore incubated cell lysates of HEK293 cells transfected with the four IL-11R variants either with PNGaseF or left them untreated. As shown in Fig. 2B, treatment with PNGaseF reduced the apparent molecular weight of IL-11R wt, IL-11R-N127Q and IL-11R-N194Q confirming that each of these variants had at least one N-glycan attached. For IL-11R-N127Q/194Q however, no shift in molecular weight was observed upon PNGaseF treatment, indicating that this IL11R mutant did not contain any N-glycans (Fig. 2B). Interestingly, PNGaseF-treatment of IL11R-N194Q resulted in a single band of the same size as the unglycosylated IL-11R, whereas for IL-11R wt and IL-11R-N127Q a second band of higher molecular weight was observed that was resistant to PNGaseF-treatment. As O-linked glycans are attached rather late in the Golgi complex, this observation suggests that IL-11R-N127Q, but not IL-11R-N194Q or IL$11 \mathrm{R}-\mathrm{N} 127 \mathrm{Q} / 194 \mathrm{Q}$, is able to reach the Golgi. 
Because all these experiments were done with transiently transfected HEK293 cells, we verified our observations with $\mathrm{Ba} / \mathrm{F} 3$-gp130 cells stably transduced with the individual IL-11R mutants. Western blot analysis of cell lysates with and without prior PNGaseF treatment confirmed our findings that Asn127 and Asn-194 were used as $\mathrm{N}$-glycosylation sites, and that no other N-glycans despite these are present on the IL-11R (Fig. 2C, D).

Lack of $\mathrm{N}$-glycosylation results in reduced cell surface expression of the $I L-11 R$

Having found that two $\mathrm{N}$-glycosylation sites are present on the IL-11R, we next analyzed whether mutation of these glycans would influence expression of the IL-11R on the cell surface. We again transiently expressed all four variants in HEK293 cells and determined cell surface levels via flow cytometry $48 \mathrm{~h}$ later. As shown in Fig. 3A, all IL-11R variants appeared at the cell surface, although in varying amounts, indicating that $\mathrm{N}$-glycosylation is important but not a prerequisite for intracellular transport and cell surface expression of the IL-11R. In order to compare the IL-11R mutants, we set the cell surface expression of IL-11R wt to $100 \%$ and calculated all other values accordingly (Fig. 3B). The N127Q mutation caused the mildest reduction of $\mathrm{IL}-11 \mathrm{R}$ at the cell surface $(60.5 \pm 12.7 \%)$, whereas $\mathrm{N} 194 \mathrm{Q}(26.0 \pm 7.2 \%)$ and N127Q/194Q $(17.3 \pm 9.0 \%)$

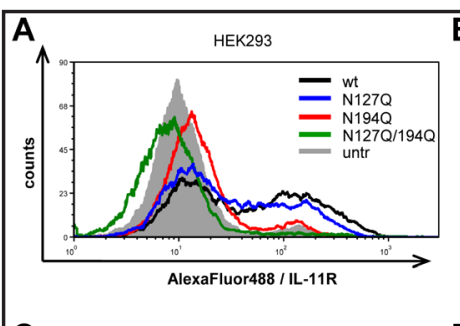

C

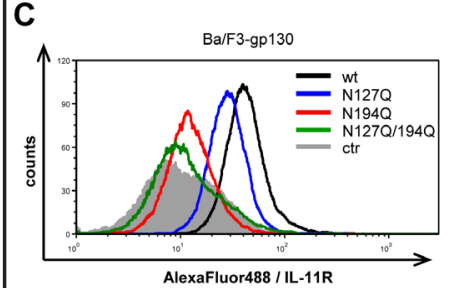

D
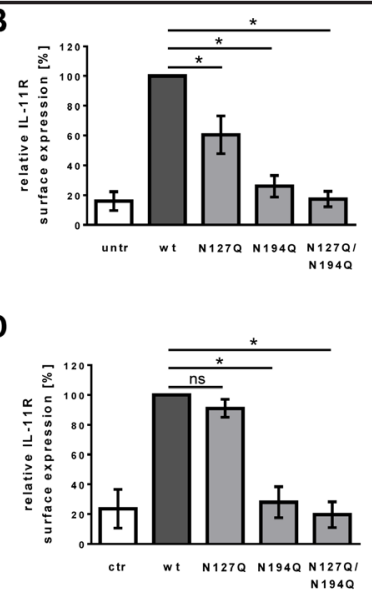

E

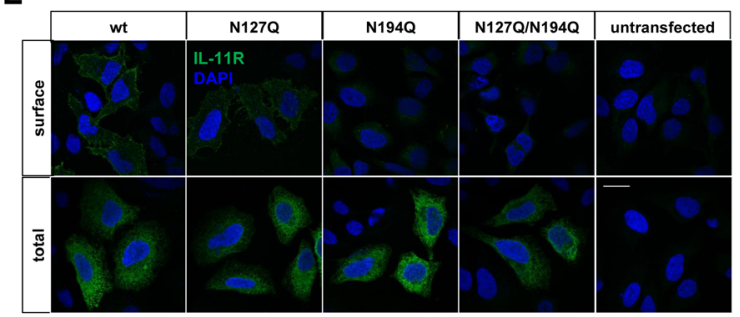

Fig. 3. Glycosylation influences the cell surface expression of the IL-11R. A, B: Plasmids coding for the indicated IL-11R variants were transiently expressed in HEK293 cells. $48 \mathrm{~h}$ after transfection cells were harvested and cell surface expression of the receptors were quantified by flow cytometry. Untransfected (untr) cells served as negative control. (A) One representative histogram is shown. (B) Quantification of the mean fluorescent intensity (mean \pm SD) normalized to the wildtype expression $(100 \%)$ of at least three independent experiments is depicted ${ }^{*}$ $=\mathrm{p}<0.05)$. C, D: Cell surface expression of the different IL-11R variants in stably transduced Ba/F3-gp130 cells was analyzed as described in panel A and B. Non-transduced Ba/F3-gp130 served as negative control (ctr). Quantification of the mean fluorescent intensity (mean \pm SD) normalized to the wildtype expression $(100 \%)$ of at least three independent experiments is depicted $\left(^{*}=p<0.05, \mathrm{~ns}=\right.$ not significant). E: HeLa cells were seeded on coverslips and transiently transfected with either IL-11R wildtype or plasmids coding for the indicated $\mathrm{N}$-glycosylation variants. $48 \mathrm{~h}$ after transfection cells were fixed and stained using a blocking solution with saponin (intracellular staining) or without saponin (surface staining). Stained slides were visualized on a confocal microscope. Scale bar: $20 \mu \mathrm{m}$. were much stronger reduced (Fig. 3B).

We further verified these results with the stably transduced Ba/F3-gp130 cell lines. Like in the HEK293 cells, all IL-11R variants reached the cell surface as determined by flow cytometry, but again to different extend (Fig. 3C). Using the same quantification strategy as before, the N127Q variant showed again the weakest reduction in cell surface appearance compared to IL-11R wt ( $91.0 \pm 6.0 \%$, Fig. 3D). In contrast, N194Q (28.0 $\pm 10.4 \%)$ and N127Q/ 


\section{Cellular Physiology and Biochemistry}

N194Q $(19.7 \pm 15.0 \%)$ led to much less IL-11R at the cell surface (Fig. 3D). In conclusion, N-glycosylation is important for intracellular transport and/ or cell surface expression of the IL-11R, and the glycan on Asn-194 appears to be more critical for this than the glycan on Asn-127.

To further strengthen this point, we transfected the IL-11R mutants in HeLa cells and analyzed their expression and fate via confocal microscopy. As shown in Fig. 3E, we could detect IL-11R wt on the cell surface of non-permeabilized HeLa cells. Abundance of IL11R-N127Q was reduced compared to the wt, and IL-11R-N194Q and IL-11RN127Q/N194Q were hardly detected at all. Intracellular total staining using permeabilized cells was performed to verify that all IL-11R variants were expressed (Fig. 3E).

\section{IL-11R mutants lacking} N194 are retained within the ER and do not reach the Golgi

The different band pattern observed for the IL11R single mutants N127Q and N194Q (Fig. 2A) suggest that $\mathrm{N194Q}$ is retained within the ER, whereas $\mathrm{N} 127 \mathrm{Q}$ is able to leave the ER and to reach the Golgi complex. In order to further strengthen this hypothesis, we transfected HeLa cells transiently with plasmids encoding IL-11R, IL-11R-N127Q, IL-11R-N194Q and IL-11R-N127Q/N194Q and visualized IL-11R expression via immune fluorescence microscopy (Fig. 4A). We costained the endogenous cis-Golgi matrix protein GM130, a well-established marker protein for the Golgi complex (Fig. 4A), and found profound overlap of GM130 with IL-11R wt (Manders' Overlap Coefficient $(\mathrm{MOC})=0.76 \pm 0.19)$ and IL-11R-N127Q $(\mathrm{MOC}=0.64 \pm 0.22)$, which was significantly lower for IL-11R-N194Q (MOC $=0.33 \pm 0.2$ ) and IL-11R-N127Q/N194Q (MOC $=0.25 \pm 0.13$, Fig. 4B). Because O-linked glycosylation occurs only in the Golgi complex and not in the ER, we incubated cell lysates of cells expressing the four IL-11R variants with

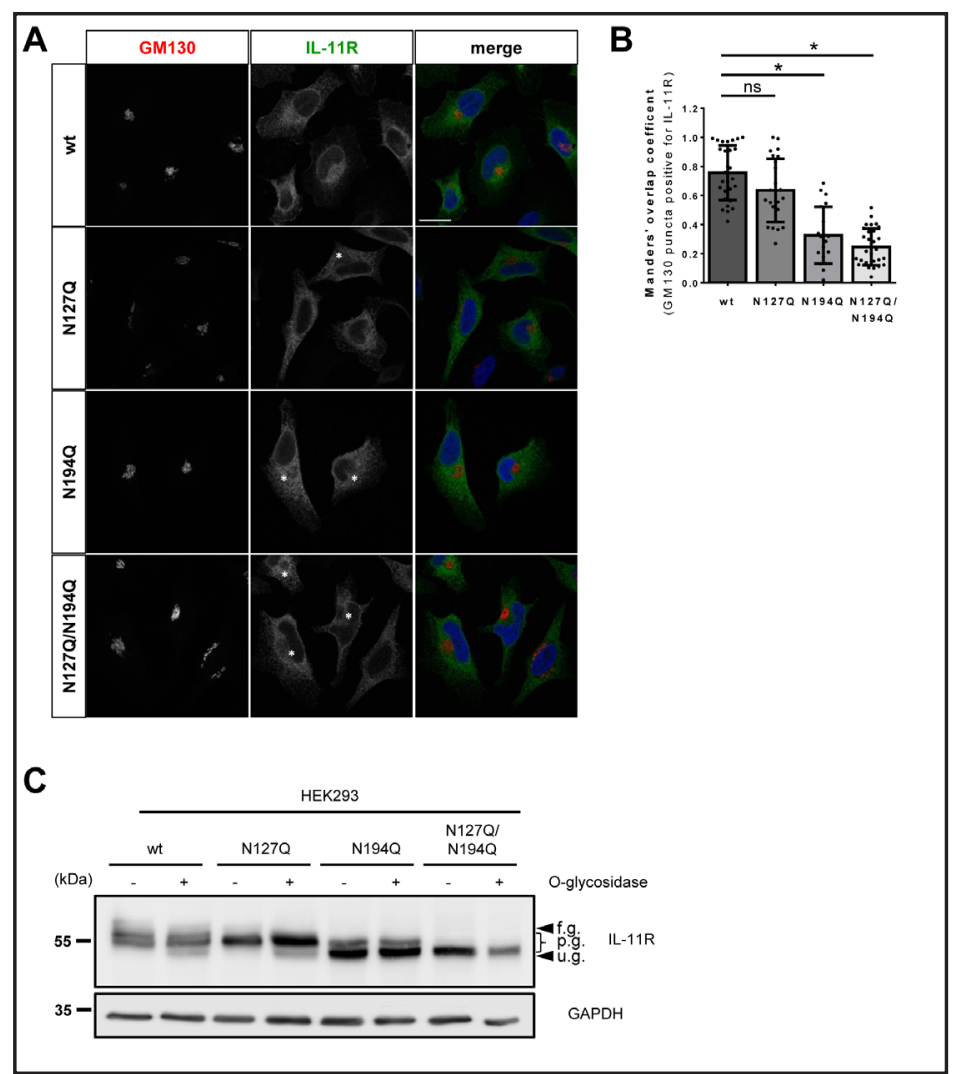

Fig. 4. Glycosylation at N194 is important for receptor export from the ER. A: HeLa cells were seeded on coverslips and transiently transfected with either IL-11R wildtype or the N-glycosylation mutants. $48 \mathrm{~h}$ after transfection cells were fixed, permeabilized and stained for overexpressed IL-11R (green) and the endogenous Golgi protein GM130 (red). Stained slides were analyzed for IL-11R and GM130 coand GM130 are marked with an asterisk. Scale bar: $20 \mu \mathrm{m}$. B: Manders' Overlap coefficient M2 for GM130 and IL-11R. Quantification of $\geq$ 18 cells from three independent experiments per IL-11R variant as depicted in $\mathrm{A}\left({ }^{*}=\mathrm{p}<0.05\right.$, ns $=$ not significant). C: HEK293 cells were prepared as described in Fig. 2B but treated with O-glycosidase over night at $37^{\circ} \mathrm{C}$. Changes in the apparent molecular weight were detected via a band shift in the Western blot. GAPDH served as control for equal protein loading. One representative blot out of three independent experiments is shown. f.g.: fully glycosylated, p.g.: partially glycosylated, u.g.: unglycosylated. localization on a confocal microscope. Cells without overlap of IL-11R 
O-glycosidase and analyzed possible alterations of the banding pattern via Western blot. As shown in Fig. 4C, treatment with O-glycosidase altered the apparent molecular weight of IL-11R wt and IL-11RN127Q, while no shift was observed for IL-11R-N194Q and IL-11R-N127Q/N194Q. This indicates that the latter two IL-11R variants do not reach the Golgi apparatus and are therefore not $\quad 0$-glycosylated. Collectively, our results show that attachment of an $\mathrm{N}$-glycan at position N194 is a prerequisite for the IL$11 \mathrm{R}$ to exit the ER and to undergo further maturation, whereas the N-glycan at N127 is dispensable in this regard.

Loss of N-linked
glycosylation does not
induce degradation
of the IL-11R via the
proteasome or the
lysosome

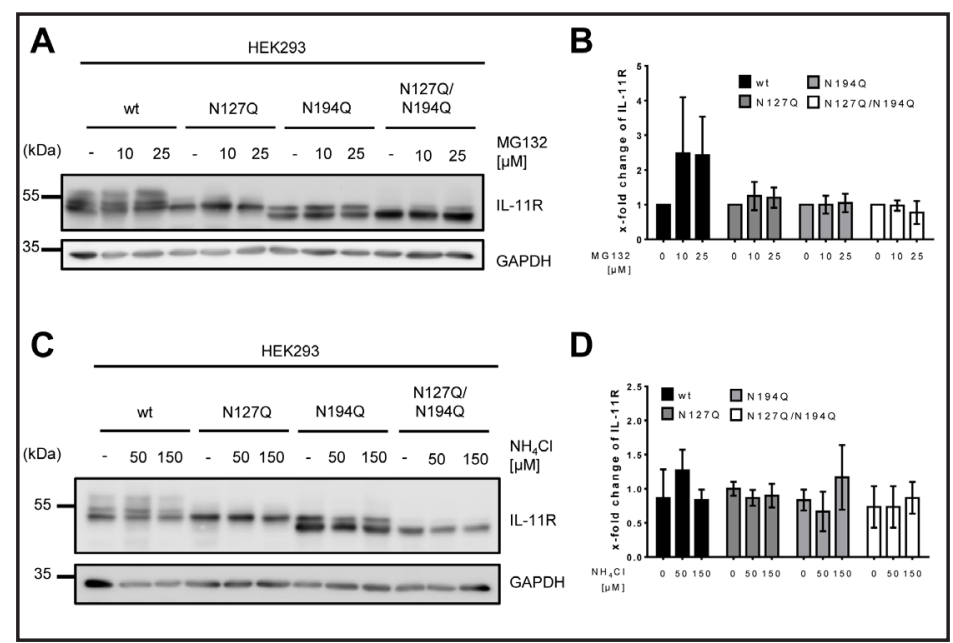

Fig. 5. Partially glycosylated or unglycosylated IL-11R is not substantially degraded via the proteasome or lysosome. A: HEK293 cells transfected with cDNA encoding the different IL-11R $\mathrm{N}$-glycosylation mutants were treated with the proteasome inhibitor MG132 (10 or $25 \mu \mathrm{M}$ ) over night. Cells were harvested afterwards and lysed in order to analyze the amount of IL-11R in the cell lysate via Western blot. B: Quantification (mean \pm SD) of the total amount of all detectable IL-11R bands per glycosylation mutant as depicted in A. The $\mathrm{x}$-fold change normalized to the untreated wildtype sample of at least three independent experiments is shown. C: HEK293 cells were prepared as described in A but treated with the lysosome inhibitor $\mathrm{NH}_{4} \mathrm{Cl}(50$ or $150 \mu \mathrm{M})$ for $8 \mathrm{~h}$. D: Quantification of the total amount of all detectable IL-11R bands normalized to the untreated wildtype sample as defined in B. GAPDH served as control for equal protein loading. One representative blot out of three independent experiments is shown.

Having shown that the IL-11R variants containing the N194Q mutation are less present at the cell surface and rather retained within the ER, we analyzed whether they are intracellularly degraded. We could recently show that C-terminal fragments of the IL-11R after ectodomain cleavage by ADAM10 are degraded via the proteasome [37]. In order to test degradation via the proteasome, we incubated transiently transfected HEK293 cells with the four IL-11R variants, added $10 \mu \mathrm{M}$ or $25 \mu \mathrm{M}$ MG132 over night or left them untreated, and analyzed IL-11R via Western blot afterwards (Fig. 5A). Interestingly, quantification of the band intensities revealed no significant increase when the cells were incubated with MG132, although we observed a tendency for the IL-11R wt (Fig. 5B). Overall, however, our results indicated that proteasomal degradation did not contribute to a major extent to the intracellular turnover of the IL-11R (Fig. 5B).

In order to analyze a possible involvement of the lysosome, we repeated the experiment described above, but added $50 \mu \mathrm{M}$ and $150 \mu \mathrm{M} \mathrm{NH}{ }_{4} \mathrm{Cl}$ for $8 \mathrm{~h}$ to the cells. This treatment increases the $\mathrm{pH}$ within the lysosomes and efficiently prevents their biological function, as we and others have shown previously [38]. However, comparable to inhibition of the proteasome, we were not able to detect a significant increase of IL-11R abundance when we prevented lysosomal degradation (Fig. 5C, D). In summary, although IL-11R-N194Q and IL-11R-N127Q/N194Q were retained within the ER compartment, we found no evidence of increased degradation of these IL-11R variants via the proteasome or the lysosome. 
$N$-glycosylation is dispensable for IL-11 induced STAT3 phosphorylation and cell proliferation

$\mathrm{Ba} / \mathrm{F} 3$ is a murine pre-B-cell line that proliferates in the presence of the cytokine IL-3 and undergoes apoptosis otherwise. However, stable expression of gp 130 and IL-11R renders them responsive towards stimulation by IL-11 $[16,19]$. We therefore used the generated $\mathrm{Ba} /$ F3-gp130 cell lines (Fig. 2C, D and 3C, D) to analyze whether N-glycosylation of the IL-11R was important for IL-11-induced signal transduction. Ba/F3-gp130-IL-11R cells were highly responsive towards stimulation by IL-11 and showed a proliferative response already at a concentration of $0.01 \mathrm{ng} / \mathrm{ml}$. Saturation was reached at a concentration of $1 \mathrm{ng} / \mathrm{ml}$, which could not be further increased by 10 or $100 \mathrm{ng} / \mathrm{ml}$ (Fig. 6A). Furthermore, phosphorylation of the transcription factor STAT3 was observed $15 \mathrm{~min}$ after stimulation with $10 \mathrm{ng} / \mathrm{ml}$ IL-11 (Fig. 6A). We observed the same when we stimulated Ba/F3-gp130-IL-11R-N127Q cells with IL-11 (Fig. 6B), despite the fact that they are having slightly reduced amounts of IL-11R at the cell surface (Fig. 3D).

Surprisingly, also Ba/F3-gp130-IL-11R-N194Q cells responded to $0.01 \mathrm{ng} / \mathrm{ml} \mathrm{IL-11} \mathrm{and}$ reached maximal proliferation at $1 \mathrm{ng} / \mathrm{ml} \mathrm{IL-11} \mathrm{(Fig.} \mathrm{6C),} \mathrm{although} \mathrm{the} \mathrm{cell} \mathrm{surface} \mathrm{expression}$ of the IL-11R was reduced by more than 70\% (Fig. 3D), and also STAT3 phosphorylation was detected after 15 min of stimulation (Fig. 6C). In contrast, we were not able to detect proliferating Ba/F3-gp130-IL-11R-N127Q/N194Q cells when $0.01 \mathrm{ng} / \mathrm{ml} \mathrm{IL-11} \mathrm{were} \mathrm{added,}$ and observed only a weak response to $1 \mathrm{ng} / \mathrm{ml}$, which slightly increased with higher amounts of IL-11 (Fig. 6D). Furthermore, the activation of STAT3 was delayed compared to the other $\mathrm{Ba} / \mathrm{F} 3$-gp130-IL-11R cell lines. This suggests that although the receptor is biologically active, its ability to induce cell proliferation is drastically impaired. A likely explanation is that a certain amount of IL-11R at the cell surface is required for optimal activation of gp130-mediated signaling, and that this critical threshold is undercut for the double mutant,

Fig. 6. N-glycosylation is not essential for IL-11R induced signaling. A-E (upper panels): Ba/F3-gp130 cells stably expressing the indicated IL$11 \mathrm{R}$ variant were incubated with increasing amounts of recombinant IL-11. After 48 h cell proliferation was measured via a cell viability assay. One representative experiment out of three is shown (mean $\pm \mathrm{SD}$, RLU: relative light units). A-E (lower panels): Stably transduced $\mathrm{Ba} / \mathrm{F} 3-$ gp130 cells were stimulated with $10 \mathrm{ng} / \mathrm{ml}$ IL-11. Cells were then harvested at different time points $(0,15,30,45$ and $60 \mathrm{~min})$ and analyzed for STAT3 phosphorylation. Total STAT3 served as control to ensure equal protein loading. One representative blot each out of three experiments with similar outcome is shown.

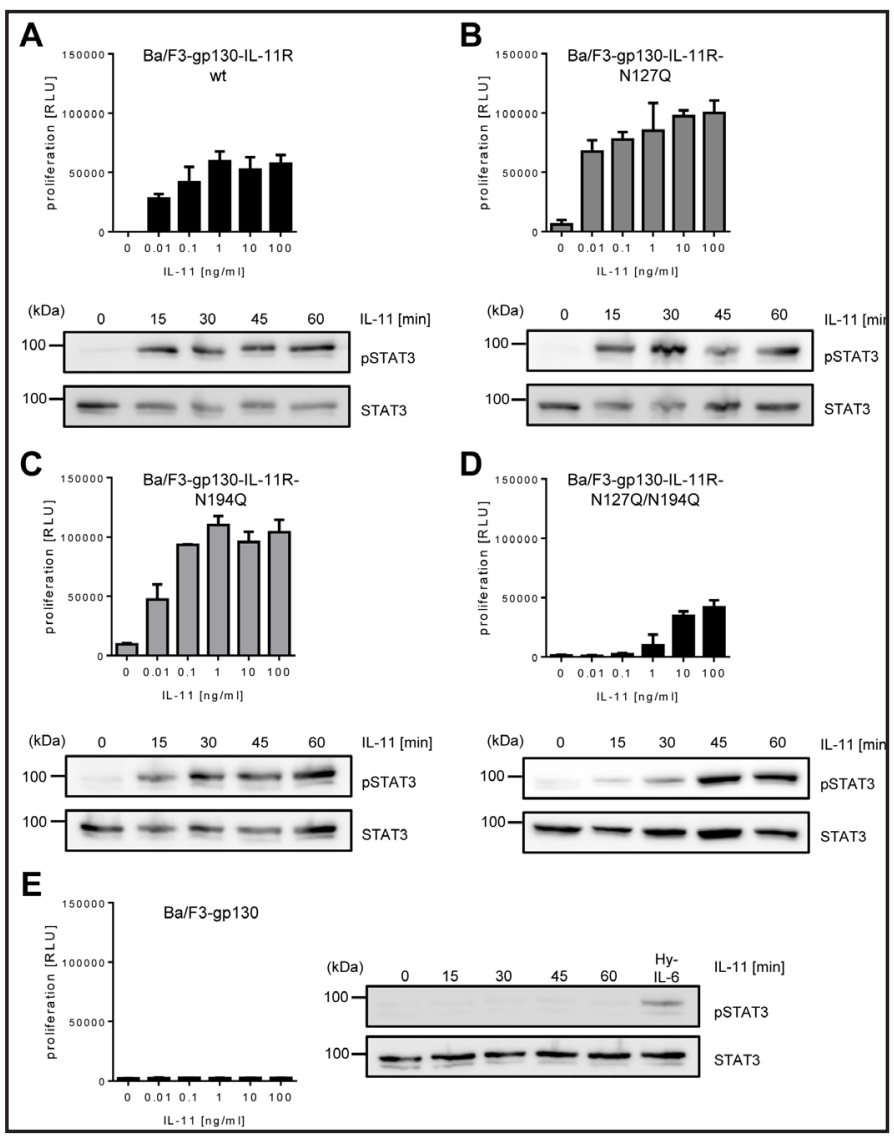


but not the single mutants N127Q and N194Q. Indeed, we did only observe marginal cell surface appearance of IL-11R-N127Q/N194Q (Fig. 3D). However, it cannot be excluded that the activation of other intracellular signaling cascades is also influenced, which might contribute to the altered cell proliferation of the Ba/F3-gp130-IL-11R-N127Q/N194Q cell line. Importantly, the parental Ba/F3-gp130 cells did not respond to IL-11 at all, confirming that the small biological response of the Ba/F3-gp130-IL-11R-N127Q/N194Q cells indeed originated from the stably expressed IL-11R variant (Fig. 6E).

\section{Inducible IL-11R shedding by ADAM10 is impaired when N-glycosylation is absent}

We have previously shown that treatment of cells with the ionophor ionomycin activates the metalloprotease ADAM10, which is able to cleave the IL-11R and thereby releasing a biologically active soluble IL-11R (sIL-11R) ectodomain $[19,20]$. Interestingly, we could recently show that an N-glycan within the D1 domain of the IL-6R acts as an exosite that regulates IL-6R proteolysis [25]. Therefore, we were curious to analyze how N-linked glycosylation influences IL-11R proteolysis and transiently expressed IL-11R wt, N127Q, N194Q and the double mutant N127Q/194Q in HEK293 cells. We stimulated the cells for 60 min with ionomycin or the solvent DMSO as control and precipitated sIL-11R from the cell supernatant for subsequent analysis via Western blot.

As shown in Fig.7A and reported previously [19], DMSO-treated HEK293 cells transfected with IL-11R released sIL-11R into the supernatant due to constitutive proteolysis, and this was increased upon treatment with ionomycin (Fig. 7A, B). In contrast, nearly no constitutive cleavage of IL-11R-N127Q and especially IL-11R-N194Q was detectable. Stimulation of ADAM10 led to proteolysis of both variants, but this was strongly reduced compared to IL11R wt (Fig. 7A, B). While this might by explained for IL-11R-N194Q due to the reduced cell surface appearance compared to IL-11R wt, expression of IL-11R-N127Q was still transported to the cell surface in significant amounts, suggesting that the N-glycan at N127 is important for ADAM10-mediated proteolysis. Surprisingly, IL-11R-N127Q/194Q was constitutively released into the cell supernatant, but no inducible shedding by ADAM10 was visible, as the amounts of sIL-11R did not increase upon ionomycin stimulation (Fig. 7A, B). Furthermore,

Fig. 7. Receptor shedding is impaired for IL-11R N-glycosylation variants. A, C: Transiently transfected HEK293 cells were stimulated for $1 \mathrm{~h}$ with $1 \mu \mathrm{M}$ of the ADAM10-activator ionomycin or for $4 \mathrm{~h}$ with $3 \mu \mathrm{M}$ of the ADAM10specific inhibitor GI254023X (GI). Soluble receptors were precipitated from the cell culture supernatant and analyzed via Western blot. Additionally, cells were lysed and also visualized via Western blotting. GAPDH served as control for equal protein loading. One representative blot out of three independent experiments is shown. B, D: Western blots from three independent experiments as described in $\mathrm{A}$ and $C$ were quantified. The amount of soluble IL-11R was normalized to the respective untreated wildtype sample (mean $\pm \mathrm{SD},{ }^{*}=\mathrm{p}<0.05$,).

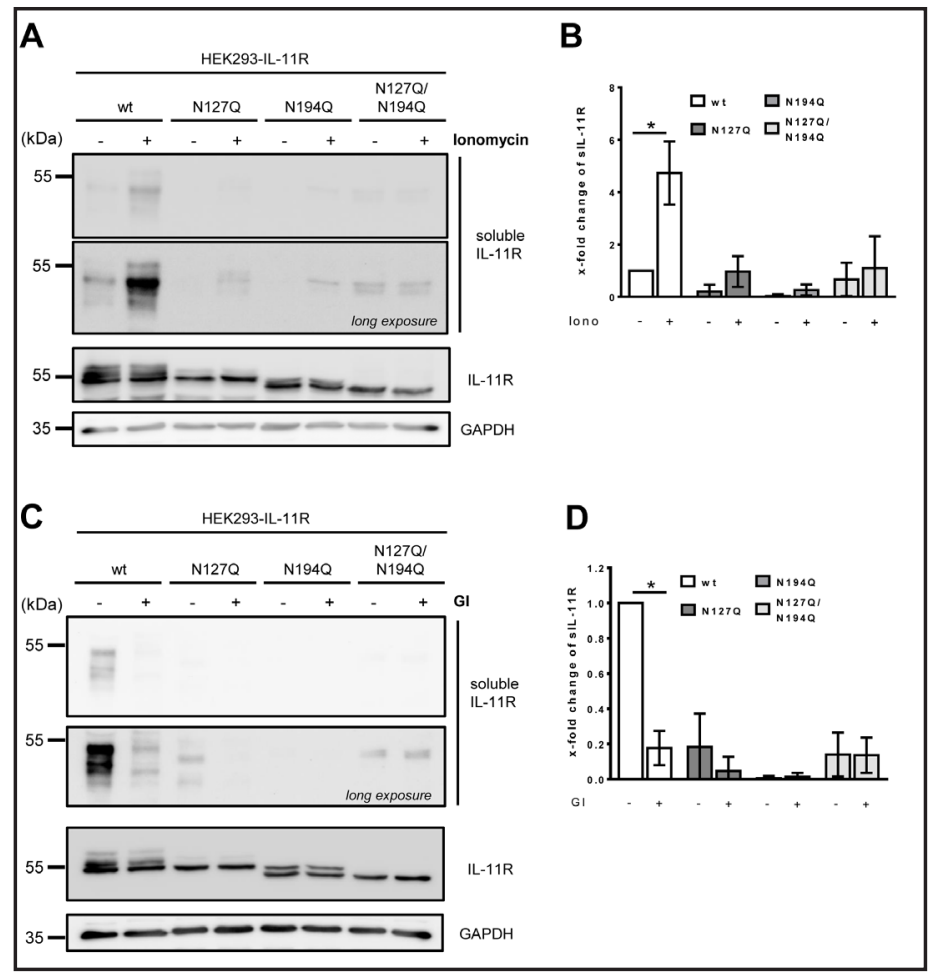




\section{Cellular Physiology Cell Physiol Biochem 2018;45:2071-2085 \\ \begin{tabular}{c|c|c|} 
DOI: 10.1159/000488044 & and Biochemistry Published online:March 12, 2018 & $\begin{array}{l}\text { O } 2018 \text { The Author(s). Published by S. Karger AG, Basel } \\
\text { www.karger.com/cpb }\end{array}$ \\
\cline { 2 - 3 }
\end{tabular}}

Agthe et al.: N-Glycosylation of the IL-11R

treatment of the HEK293 cells for $4 \mathrm{~h}$ with GI254023X (GI), a specific inhibitor of ADAM10, strongly diminished constitutive cleavage of the IL-11R wt, and completely abrogated the constitutive processing of IL-11R-N127Q further providing evidence that ADAM10 is involved in this process (Fig. 7C, D). We could still not detect any constitutive cleavage of IL11R-N194Q even after $4 \mathrm{~h}$ (Fig. 7C, D). Interestingly, the release of the double mutant IL-11RN127Q/N194Q was completely unaffected by GI-treatment, confirming that ADAM10 is not involved (Fig. 7C, D). It is possible that IL-11R-N127Q/N194Q is either released by a second protease which is not inhibited by GI, or that the IL-11R variant is released by a completely different mechanism, e.g. on microvesicles.

\section{Discussion}

Cytokines of the IL- 6 family play important roles in homeostasis and are key molecules in inflammatory diseases. Importantly, the expression of their receptors dictates which cells can be activated by the secreted cytokines, and especially the cellular expression pattern of the unique individual IL-11 and IL- $6 \alpha$-receptors is fundamental for the specific activities of the two cytokines, since the signal-transducing $\beta$-receptor gp130 is expressed ubiquitously.

While the biological functions and properties of the IL-6R are well studied and understood (reviewed e.g. in $[21,39,40]$ ), rather less attention has been paid to the IL-11R. We have recently shown that the IL-11R has a similar modular organization like the IL-6R [16], and while glycosylation of the IL-6R is described in much detail [25, 41, 42], functional roles for glycans in IL-11 biology have not been analyzed so far. While the IL-6R contains five N-glycosylation sites, two located in the D1 and D3 domains each and one within the stalk region, the amino acid sequence of the IL-11R contains only the two sequons at N127 and N194 within its D2 domain that could be potentially used for N-glycosylation [35]. Here, we show via different experimental approaches that both sequons are indeed used for $\mathrm{N}$-linked glycosylation, and that besides these no additional $\mathrm{N}$-linked glycans are attached to the IL-11R. Furthermore, we show evidence that the IL-11R is additionally 0-glycosylated. It is currently unclear why the number and the distribution of the $\mathrm{N}$-glycans is different between the IL-6R and the IL-11R, which are always considered as rather similar in function and structure. Strikingly, while the D2 domain of the IL-6R is not glycosylated [25], both $\mathrm{N}$-glycans of the entire IL-11R ectodomain are located at D2.

Although mutation of both $\mathrm{N}$-glycan sequons resulted in strongly reduced cell surface appearance of the IL-11R, the few receptors that reached the plasma membrane were still biologically active and able to induce STAT3 phosphorylation and proliferation of the respective $\mathrm{Ba} / \mathrm{F} 3$-gp130 cell line, although way less efficiently than the wildtype IL-11R or the single mutant. A similar effect has been demonstrated for gp130 devoid of all N-glycan sites [24]. On the contrary, although the cell surface expression of an IL-6R mutant lacking all five N-glycans was reduced, its biological activity was indistinguishable from its wildtype counterpart [25]. These findings suggest that the stability of the IL-6R depends less on the glycosylation compared to the IL-11R, although the molecular reason for this discrepancy is not known.

Another important finding of this study is the differential importance of the two $\mathrm{N}$-glycan sequons for intracellular transport and thus maturation of the IL-11R. Mutation of N127 and thus loss of the N-glycan at this position is largely tolerated by the protein, which is able to leave the ER, travel through the Golgi complex and reach the cell surface. In contrast, mutation of the sequon containing N194, either alone or in combination with N127, prevents ER exit of the majority of the IL-11R. This results in reduced co-localization with the Golgi complex marker GM130, a lack of O-glycosylation and drastically reduced cell surface appearance of the IL-11R mutants. Differential N-glycosylation patterns have been described, e.g. for several tumor tissues $[43,44]$, and appear to be present even on a subcellular level [45]. Nevertheless, it is tempting to speculate that the N-linked glycan at N194 is important for correct folding of the D2 domain of the IL-11R and the subsequent exit 
of the ER, while the glycan at N127 is dispensable for this process.

Proteolysis by ADAM proteases is believed to occur at the plasma membrane, and it is therefore not surprising to detect reduced shedding of the three glycan mutants given their reduced cell surface expression. Although the reduction of sIL-11R generated by ADAM10 appears to be stronger as anticipated by the lower cell surface expression, it is difficult to convincingly determine this undoubtedly. The results, however, exclude a major direct role of the glycans for processing of the IL-11R by ADAM10. Importantly, in contrast to the IL-6R [25], we could not detect increased shedding of one of the mutant receptors, which indicates that $\mathrm{N}$-glycans have different functional roles for IL-6R and IL-11R. Intriguingly, the double mutant IL-11R-N127Q/N194Q was not shed by ADAM10, but rather constitutively released from the cells, pointing towards a different mechanism. While we cannot rule out the involvement of another protease, we could recently show that the IL-6R can be found as a full-length protein on circulating microvesicles [46], and it might well be that the IL-11R mutant described here is secreted via a similar pathway.

In summary, we show that the D2 domain of the IL-11R contains two N-linked glycans, which are dispensable for its biological activity, but differentially control maturation and intracellular trafficking of the receptor.

\section{Acknowledgements}

The authors thank Alyn Gerneth for excellent technical assistance.

This work was funded by grants from the Deutsche Forschungsgemeinschaft, Bonn, Germany (SFB877 project A10 to C.G.) and by the Cluster of Excellence 'Inflammation at Interfaces'.

\section{Disclosure Statement}

No conflict of interests exist.

\section{References}

1 Ohtsubo K, Marth JD: Glycosylation in Cellular Mechanisms of Health and Disease. Cell 2006;126:855-867.

- Xu C, Ng DTW: Glycosylation-directed quality control of protein folding. Nat Rev Mol Cell Biol 2015;16:742752.

-3 Apweiler R, Hermjakob H, Sharon N: On the frequency of protein glycosylation, as deduced from analysis of the SWISS-PROT database. Biochim Biophys Acta 1999;1473:4-8.

-4 Moremen KW, Tiemeyer M, Nairn AV: Vertebrate protein glycosylation: diversity, synthesis and function. Nat Rev Mol Cell Biol 2012;13:448-462.

-5 Van den Steen P, Rudd PM, Dwek RA, Opdenakker G: Concepts and principles of O-linked glycosylation. Crit Rev Biochem Mol Biol 1998;33:151-208.

6 Garbers C, Hermanns H, Schaper F, Müller-Newen G, Grötzinger J, Rose-John S, Scheller J: Plasticity and cross-talk of Interleukin 6-type cytokines. Cytokine Growth Factor Rev 2012;23:85-97.

7 Garbers C, Scheller J: Interleukin-6 and interleukin-11: same same but different. Biol Chem 2013;394:11451161.

8 Ren C, Chen Y, Han C, Fu D, Chen H: Plasma interleukin-11 (IL-11) levels have diagnostic and prognostic roles in patients with pancreatic cancer. Tumor Biol 2014;35:11467-11472.

-9 Ren L, Wang X, Dong Z, Liu J, Zhang S: Bone metastasis from breast cancer involves elevated IL-11 expression and the gp130/STAT3 pathway. Med Oncol 2013;30:634.

10 Chen CC, Wang SS, Lu RH, Chang FY, Lee SD: Serum interleukin 10 and interleukin 11 in patients with acute pancreatitis. Gut 1999;45:895-899. 


\section{Cellular Physiology Cell Physiol Biochem 2018;45:2071-2085

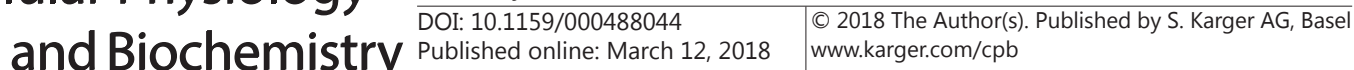

Agthe et al.: N-Glycosylation of the IL-11R

11 Trontzas P, Kamper EF, Potamianou A, Kyriazis NC, Kritikos H, Stavridis J: Comparative study of serum and synovial fluid interleukin-11 levels in patients with various arthritides. Clin Biochem 1998;31:673-679.

-12 Winship A, Dimitriadis E: Interleukin-11 induces preterm birth and modulates decidual inflammasome gene expression in mice. Placenta 2017;50:99-101.

13 Winship AL, Koga K, Menkhorst E, Van Sinderen M, Rainczuk K, Nagai M, Cuman C, Yap J, Zhang J-GG, Simmons D, Young MJ, Dimitriadis E: Interleukin-11 alters placentation and causes preeclampsia features in mice. Proc Natl Acad Sci U S A 2015;112:15928-15933.

14 Putoczki T, Thiem S, Loving A, Busuttil R, Wilson N, Ziegler P, Nguyen P, Preaudet A, Farid R, Edwards K, Boglev Y, Luwor R, Jarnicki A, Horst D, Boussioutas A, Heath J, Sieber O, Pleines I, Kile B, Nash A, Greten F, McKenzie B, Ernst M: Interleukin-11 Is the Dominant IL-6 Family Cytokine during Gastrointestinal Tumorigenesis and Can Be Targeted Therapeutically. Cancer Cell 2013;24:257-271.

15 Putoczki TL, Ernst M: IL-11 signaling as a therapeutic target for cancer. Immunotherapy 2015;7:441-453.

-16 Nitz R, Lokau J, Aparicio-Siegmund S, Scheller J, Garbers C: Modular organization of Interleukin-6 and Interleukin-11 $\alpha$-receptors. Biochimie 2015;119:175-182.

17 Garbers C, Aparicio-Siegmund S, Rose-John S: The IL-6/gp130/STAT3 signaling axis: recent advances towards specific inhibition. Curr Opin Immunol 2015;34:75-82.

18 Monhasery N, Moll J, Cuman C, Franke M, Lamertz L, Nitz R, Gorg B, Häussinger D, Lokau J, Floss DM, Piekorz R, Dimitriadis E, Garbers C, Scheller J: Transcytosis of IL-11 and Apical Redirection of gp130 Is Mediated by IL-11alpha Receptor. Cell Rep 2016;16:1067-1081.

19 Lokau J, Nitz R, Agthe M, Monhasery N, Aparicio-Siegmund S, Schumacher N, Wolf J, Möller-Hackbarth K, Waetzig GH, Grötzinger J, Müller-Newen G, Rose-John S, Scheller J, Garbers C: Proteolytic Cleavage Governs Interleukin-11 Trans-signaling. Cell Rep 2016;14:1761-1773.

-20 Lokau J, Wandel M, Garbers C: Enhancing Interleukin-6 and Interleukin-11 receptor cleavage. Int J Biochem Cell Biol 2017;85:6-14.

-21 Lokau J, Agthe M, Garbers C: Generation of Soluble Interleukin-11 and Interleukin-6 Receptors: A Crucial Function for Proteases during Inflammation. Mediators Inflamm 2016;2016:1785021.

22 Agthe M, Garbers Y, Putoczki T, Garbers C: Interleukin-11 classic but not trans-signaling is essential for fertility in mice. Placenta 2017;57:13-16.

23 Balic JJ, Garbers C, Rose-John S, Yu L, Jenkins BJ: Interleukin-11-driven gastric tumourigenesis is independent of trans-signalling. Cytokine 2017;92:118-123.

24 Waetzig GH, Chalaris A, Rosenstiel P, Suthaus J, Holland C, Karl N, Vallés Uriarte L, Till A, Scheller J, Grötzinger J, Schreiber S, Rose-John S, Seegert D: N-linked glycosylation is essential for the stability but not the signaling function of the interleukin-6 signal transducer glycoprotein 130. J Biol Chem 2010;285:17811789.

25 Riethmueller S, Somasundaram P, Ehlers JC, Hung C-WW, Flynn CM, Lokau J, Agthe M, Düsterhöft S, Zhu Y, Grötzinger J, Lorenzen I, Koudelka T, Yamamoto K, Pickhinke U, Wichert R, Becker-Pauly C, Rädisch M, Albrecht A, Hessefort M, Stahnke D, Unverzagt C, Rose-John S, Tholey A, Garbers C: Proteolytic Origin of the Soluble Human IL-6R In vivo and a Decisive Role of N-Glycosylation. PLoS Biol 2017;15:e2000080

-26 Ishino T, Economou NJ, McFadden K, Zaks-Zilberman M, Jost M, Baxter S, Contarino MR, Harrington AE, Loll PJ, Pasut G, Lievens S, Tavernier J, Chaiken I: A protein engineering approach differentiates the functional importance of carbohydrate moieties of interleukin-5 receptor $\alpha$. Biochemistry 2011;50:7546-7556.

-27 Slieker LJ, Lane MD: Post-translational processing of the epidermal growth factor receptor. Glycosylationdependent acquisition of ligand-binding capacity. J Biol Chem 1985;260:687-690.

28 Ding DXH, Vera JC, Heaney ML, Golde DW: N-glycosylation of the human granulocyte-macrophage colonystimulating factor receptor $\alpha$ subunit is essential for ligand binding and signal transduction. J Biol Chem 1995;270:24580-24584.

-29 Zhou H, Tai HH: Characterization of recombinant human CXCR4 in insect cells: role of extracellular domains and N-glycosylation in ligand binding. Arch Biochem Biophys 1999;369:267-276.

-30 Gearing DP, Ziegler SF, Comeau MR, Friend D, Thoma B, Cosman D, Park L, Mosley B: Proliferative responses and binding properties of hematopoietic cells transfected with low-affinity receptors for leukemia inhibitory factor, oncostatin M, and ciliary neurotrophic factor. Proc Natl Acad Sci U S A 1994;91:11191123. 


\section{Cellular Physiology Cell Physiol Biochem 2018;45:2071-2085

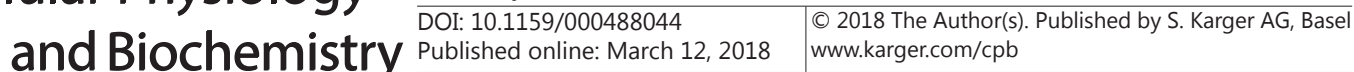

Agthe et al.: N-Glycosylation of the IL-11R

-31 Fischer M, Goldschmitt J, Peschel C, Brakenhoff JP, Kallen KJ, Wollmer A, Grötzinger J, Rose-John S: I. A bioactive designer cytokine for human hematopoietic progenitor cell expansion. Nat Biotechnol 1997;15:142-145.

32 Wang XM, Wilkin JM, Boisteau O, Harmegnies D, Blanc C, Vandenbussche P, Montero-Julian FA, Jacques Y, Content J: Engineering and use of (32)P-labeled human recombinant interleukin-11 for receptor binding studies. Eur J Biochem 2002;269:61-68.

-33 Suthaus J, Tillmann A, Lorenzen I, Bulanova E, Rose-John S, Scheller J: Forced homo- and heterodimerization of all gp130-type receptor complexes leads to constitutive ligand-independent signaling and cytokine-independent growth. Mol Biol Cell 2010;21:2797-2807.

-34 Putoczki TL, Dobson RCJ, Griffin MDW: The structure of human interleukin-11 reveals receptor-binding site features and structural differences from interleukin-6 Acta Crystallogr D Biol Crystallogr 2014;70:22772285.

-35 Chérel M, Sorel M, Lebeau B, Dubois S, Moreau JF, Bataille R: Molecular cloning of two isoforms of a receptor for the human hematopoietic cytokine interleukin-11 Blood 1995;86:2534-2540.

-36 Lebeau B, Montero Julian FA, Wijdenes J, Müller-Newen G, Dahmen H, Chérel M, Heinrich PC, Brailly H, Hallet MM, Godard A, Minvielle S, Jacques Y: Reconstitution of two isoforms of the human interleukin-11 receptor and comparison of their functional properties. FEBS Lett 1997;407:141-147.

37 Lokau J, Flynn CM, Garbers C: Cleavage of the Interleukin-11 receptor induces processing of its C-terminal fragments by the gamma-secretase and the proteasome. Biochem Biophys Res Commun 2017;491:296302.

-38 Lorenzen I, Lokau J, Korpys Y, Oldefest M, Flynn CM, Künzel U, Garbers C, Freeman M, Grötzinger J, Düsterhöft S: Control of ADAM17 activity by regulation of its cellular localisation. Sci Rep 2016;6:35067.

-39 Chalaris A, Garbers C, Rabe B, Rose-John S, Scheller J: The soluble Interleukin 6 receptor: generation and role in inflammation and cancer. Eur J Cell Biol 2011;90:484-494.

-40 Lokau J, Agthe M, Flynn CM, Garbers C: Proteolytic control of Interleukin-11 and Interleukin-6 biology. Biochim Biophys Acta 2017;1864:2105-2117.

-41 Cole AR, Hall NE, Treutlein HR, Eddes JS, Reid GE, Moritz RL, Simpson RJ: Disulfide bond structure and N-glycosylation sites of the extracellular domain of the human interleukin-6 receptor. J Biol Chem 1999;274:7207-7215.

42 Müllberg J, Oberthür W, Lottspeich F, Mehl E, Dittrich E, Graeve L, Heinrich P, Rose-John S: The soluble human IL-6 receptor. Mutational characterization of the proteolytic cleavage site. J Immunol 1994;152:4958-4968.

43 Kuzmanov U, Jiang N, Smith CR, Soosaipillai A, Diamandis EP: Differential N-glycosylation of kallikrein 6 derived from ovarian cancer cells or the central nervous system. Mol Cell Proteomics 2009;8:791-798.

44 Ruhaak LR, Taylor SL, Stroble C, Nguyen UT, Parker EA, Song T, Lebrilla CB, Rom WN, Pass H, Kim K, Kelly K, Miyamoto S: Differential N-Glycosylation Patterns in Lung Adenocarcinoma Tissue. J Proteome Res 2015;14:4538-4549.

45 Lee LY, Lin CH, Fanayan S, Packer NH, Thaysen-Andersen M: Differential site accessibility mechanistically explains subcellular-specific N-glycosylation determinants. Front Immunol 2014;5:404.

-46 Schumacher N, Meyer D, Mauermann A, von der Heyde J, Wolf J, Schwarz J, Knittler K, Murphy G, Michalek M, Garbers C, Bartsch JWW, Guo S, Schacher B, Eickholz P, Chalaris A, Rose-John S, Rabe B: Shedding of Endogenous Interleukin-6 Receptor (IL-6R) Is Governed by A Disintegrin and Metalloproteinase (ADAM) Proteases while a Full-length IL-6R Isoform Localizes to Circulating Microvesicles. J Biol Chem 2015;290:26059-26071. 